\title{
Radiation Dose Optimization in Pediatric Chest CT: Major Indicators of Dose Exposure in 1695 CT Scans over Seven Years
}

\author{
Optimierung der Strahlendosis in der Thorax-CT des Kindes: \\ Einflussfaktoren der Strahlenexposition aus 1695 CT- \\ Untersuchungen in sieben Jahren
}

\section{Authors}

Michael Esser, Sabine Hess, Matthias Teufel, Mareen Sarah Kraus, Sven Schneeweiß, Sergios Gatidis, Juergen F Schaefer, Ilias Tsiflikas

\section{Affiliation}

Radiology, Eberhard Karls University of Tuebingen, Tuebingen, Germany

Key words

thorax, CT-spiral, radiation safety

received 30.09.2017

accepted 24.04.2018

Bibliography

DOI https://doi.org/10.1055/a-0628-7222

Published online: 11.10.2018

Fortschr Röntgenstr 2018; 190: 1131-1140

(C) Georg Thieme Verlag KG, Stuttgart · New York

ISSN 1438-9029

Correspondence

Dr. Michael Esser

Radiology, Eberhard Karls University of Tuebingen, Hoppe-Seyler-Straße 3, 72074 Tuebingen, Germany

Tel.: ++49/70 71/2986677

michael.esser@med.uni-tuebingen.de

\section{ZUSAMMENFASSUNG}

Ziel Analyse möglicher Einflussfaktoren auf die Strahlenexposition bei der Thorax-CT des Kindes unter Anwendung verschiedener Methoden der Dosisoptimierung und Bestimmung von Kenngrößen für die Dosisentwicklung.

Material und Methoden In dieser retrospektiven Studie an einer Klinik der Maximalversorgung einschließlich einer Abteilung für Kinderradiologie wurden 1695 Thorax-CTs von 768 Patienten (mittleres Alter, 10 Jahre; Spannweite, zwei Tage bis 17,9 Jahre) analysiert. Volumen-CT-Dosisindex, effektive Dosis und size-specific dose estimate, automatische Dosismodulation (AEC), sowie High-Pitch-Protokolle (Pitch $\geq 3,0$ ) wurden mittels univariater Analyse ausgewertet. Die Bildqualität von Niedrigdosis-Untersuchungen wurde zu Protokollen mit höherer Dosis mittels „Non-Inferiority“-Analyse verglichen.

Ergebnisse Die medianen Dosiswerte sanken pro Jahr um durchschnittlich $12 \%$. Untersuchungen mit High-Pitch-Modus $(n=414)$ lieferten geringere Dosiswerte $(p<0,001)$. In nativen
Untersuchungen mit AEC ergaben sich höhere Dosiswerte im Vergleich zu Untersuchungen mit manuell festgelegten Parametern $(p<0,001)$. In kontrastangehobenen CTs erzielte die AEC nur bei Patienten über 16 Jahren signifikant niedrigere Dosiswerte $(p=0,04)$. Im Alter von 6 bis 15 Jahren waren die Dosiswerte mit AEC höher $(p<0,001)$. Die diagnostische Bildqualität von Niedrigdosis-Untersuchungen war den CTs mit höherer Dosis nicht unterlegen $(2,18$ vs. 2,14$)$.

Schlussfolgerung Im letzen Jahrzehnt wurden die Dosiswerte der Thorax-CT ohne Verlust der Bildqualität reduziert. High-Pitch-Protokolle sind in diesem Zusammenhang ein unabhängiger Einflussfaktor. Die Dosisreduktion mittels AEC war beschränkt und nur für Patienten über 16 Jahren relevant.

\section{Kernaussagen}

- Im letzten Jahrzehnt konnte die Strahlendosis der Thorax-CT bei Kindern reduziert werden.

- Die Untersuchung mit hohen Pitch-Werten ist ein unabhängiger Faktor der Dosisoptimierung.

- Die Dosisreduktion der AEC ist beschränkt und nur für ältere Kinder relevant.

\section{ABSTRACT}

Purpose To analyze possible influencing factors on radiation exposure in pediatric chest CT using different approaches for radiation dose optimization and to determine major indicators for dose development.

Materials and Methods In this retrospective study at a clinic with maximum care facilities including pediatric radiology, 1695 chest CT examinations in 768 patients (median age: 10 years; range: 2 days to 17.9 years) were analyzed. Volume CT dose indices, effective dose, size-specific dose estimate, automatic dose modulation (AEC), and high-pitch protocols (pitch $\geq 3.0$ ) were evaluated by univariate analysis. The image quality of low-dose examinations was compared to higher dose protocols by non-inferiority testing.

Results Median dose-specific values annually decreased by an average of $12 \%$. High-pitch mode $(n=414)$ resulted in lower dose parameters $(\mathrm{p}<0.001)$. In unenhanced CT, AEC delivered higher dose values compared to scans with fixed parameters $(p<0.001)$. In contrast-enhanced $C T$, the use of AEC yielded a 
significantly lower radiation dose only in patients older than 16 years $(p=0.04)$. In the age group 6 to 15 years, the values were higher $(p<0.001)$. The diagnostic image quality of lowdose scans was non-inferior to high-dose scans (2.18 vs. 2.14). Conclusion Radiation dose of chest CT was reduced without loss of image quality in the last decade. High-pitch scanning was an independent factor in this context. Dose reduction by AEC was limited and only relevant for patients over 16 years.

\section{Key Points}

- The radiation dose of pediatric chest CT was reduced in the last decade.
- High-pitch scanning is an independent factor of dose optimization.

- Dose reduction by AEC is limited and only relevant for older children.

\section{Citation Format}

- Esser M, Hess S, Teufel M et al. Radiation Dose Optimization in Pediatric Chest CT: Major Indicators of Dose Exposure in 1695 CT Scans over Seven Years. Fortschr Röntgenstr 2018; 190: 1131-1140

\section{Introduction}

Multidetector computed tomography (MDCT) has become an essential technique in daily practice [1, 2]. Over the years, CT imaging essentially accounted for a trend of increasing medical radiation exposure $[3,4]$. The use of CT in pediatric patients has been among the most rapidly increasing diagnostic procedures [5]. As $\mathrm{CT}$ involves significantly higher radiation doses than conventional $\mathrm{X}$-ray, possible radiation-induced side effects have to be considered [6]. In a pediatric setting, the high radio-sensitivity of the developing tissues and the remaining years of life in which cancer might occur underline the significance of an effective patient care program with careful management of pediatric patient dose. Several cohort studies have predicted an increased cancer risk associated with CT scans, particularly concerning leukemia and also some solid cancer types [2, $7-10]$.

In this context, the gradually increasing awareness of radiation exposure, mainly from CT examinations, has motivated manufacturers to develop techniques to manage radiation dose [11]. In this context, optimized protocols for routine CT examinations, state-of-the-art technique and the indication for $\mathrm{CT}$ in the light of possible adverse effects are of special concern [2]. Different methods of reducing radiation dose have been developed in recent years, such as low dose protocols [12] with automated attenuation-based tube current modulation and tube voltage selection $[13,14]$ or the use of tin filtration [15]. Moreover, reconstruction methods - like iterative algorithms [16] - and high-pitch technique have been evaluated in several studies [17 - 19]. In our study, iterative reconstruction was not applied. However, state-ofthe-art CT scanners with well managed dual-source CT protocols (e. g. low kV protocols) and, to a lesser degree, the corresponding techniques of dose reduction (e.g. iterative reconstruction) were not widely available in routine clinical practice for the examination of pediatric patients, especially until the middle of the 2010's [20]. We believe that our study is still relevant for wide ranges of pediatric examinations worldwide, where these technological innovations are not available.

The aim of this retrospective observational study was to identify trends in state-of-the-art pediatric CT concerning radiation dose management in a hospital with maximum care facilities including a specialized pediatric radiology department.

\section{Materials and methods}

All CT examinations were performed in a single center with a board-certified division of pediatric radiology in the period from January 2007 to March 2014. The inclusion criterion was clinically indicated $\mathrm{CT}$ including the chest at the age of less than 18 years.

\section{Patient characteristics}

1695 CT examinations were performed in 768 patients (mean age \pm standard deviation, $9.8 \pm 5.6$ years; median and $95 \%$ confidence level, 10.4 years [9.6; 10.1]; 1004 male (10.1 years $\pm 5.6 ; 10.4$ years, $[9.8 ; 10.5])$ and 691 female $(9.4$ years $\pm 5.5 ; 10.2$ years, $[9.0 ; 9.8]))$. Based on the relation between children age and body stature [21] and according to the diagnostic reference levels of the Federal Office for Radiation Protection [22], patients were divided into the following age groups: neonates $(n=16), \geq 1$ month and $\leq 1$ year (infants; $n=195), 2-5$ years $(n=285), 6-10$ years $(n=405), 11-15$ years $(n=531), 16-17$ years $(n=263)$. Because of the small sample size, neonates were excluded from the subgroup analyses. The underlying indications for performing CT are listed in $>$ Table 1. As $>$ Fig. 1 illustrates, the number of CT examinations did not change significantly in the observation time.

\section{CT devices}

Six different CT scanners (Siemens Healthcare $\mathrm{GmbH}$, Forchheim, Germany.; $>$ Table 2), each in line with the currently available CT generation, were used in our study, depending on the date of implementation in our institution ( $\triangleright$ Fig. 2): Somatom Sensation 64 (05/2007 - 03/2014; $42 \%$ of all examinations), Somatom Definition Flash (09/2010 - 03/2014; 26\%), Somatom Sensation Open (01/2007 - 09/2009; $23 \%)$, Somatom Definition (01/2007-05/ 2010; $7.5 \%)$, Somatom Definition AS+ (10/2009-07/2013; $1 \%)$, Somatom Sensation 16 (09/2008-04/2013; 0.5\%).

\section{CT examination}

All procedures were in accordance with the 1964 Helsinki declaration and its later amendments or comparable ethical standards. For this retrospective study formal consent was not required. All acquisitions were performed with specific pediatric $\mathrm{CT}$ protocols determined from the division of pediatric radiology. Scan parameters were adapted to patient body weight and age. The CT set- 
- Table 1 Indications for chest CT scans.

- Tab. 1 Indikationen für Thorax-CTs.

\section{indication}

number (n)

\begin{tabular}{|l|l|}
\hline infection process & 852 \\
\hline monitoring of progress or response under therapy & 239 \\
\hline non-malignant and non-infectious processes & 205 \\
\hline cardiac and vascular imaging & 149 \\
\hline staging & 147 \\
\hline follow-up & 40 \\
\hline trauma management & 21 \\
\hline
\end{tabular}

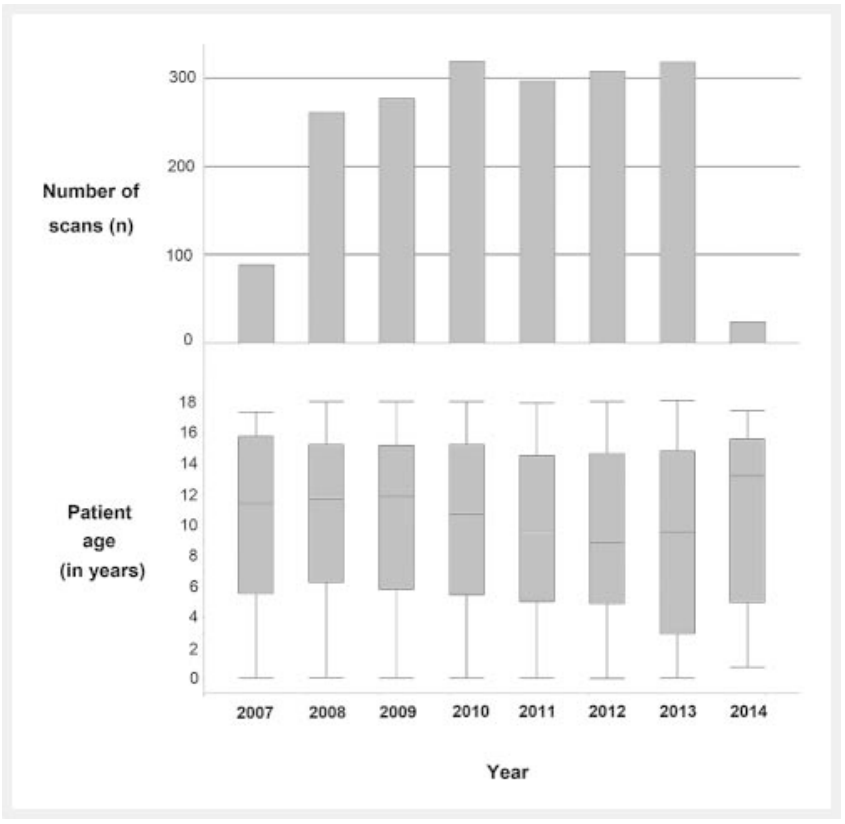

- Fig. 1 Number of scans and patient age per year. Number of scans performed $(n)$ and boxplots of patient age (in years) in the different years of the observation time. It should be noticed that in the year 2014 only three months were included in the study. In the year 200779 CTs were performed. However, the number of scans remains above 200 examinations per year in the main years of observation from 2008 to 2013. The mean patient age decreased from 10.5 to 9.1 years in 2013 .

- Abb. 1 Untersuchungszahlen und Patientenalter pro Jahr. Anzahl der durchgeführten Untersuchungen (n) und Boxplots des Patientenalters (in Jahren) in den verschiedenen Jahres des Beobachtungszeitraums. Dabei ist zu bemerken, dass im Jahr 2014 nur drei Monate in die Studie eingeschlossen wurden. Im Jahr 2007 wurden 79 CT-Untersuchungen durchgeführt. In den Hauptjahren des Beobachtungszeitraums von 2008 bis 2013 verbleibt die Anzahl der durchgeführten Untersuchungen über 200 pro Jahr. Das mittlere Patientenalter sank von 10.5 auf 9.1 Jahre im Jahr 2013. tings followed the current standard operating procedures at the time of the examination. Accordingly, parameters also depended on the technical conditions present at the time of the scan (as mentioned above; $\mathrm{CT}$ devices).

lomeprol (Imeron $400^{\circledR}$ ) or lopromid (Ultravist $370^{\circledR}$, both Bayer Healthcare Deutschland, Leverkusen, Germany) were applied as intravenous contrast agent, either via a manual injection (body weight $<10 \mathrm{~kg}$ ) or by a dual head power injection device. The injected volume was adjusted to the patients' body weight. The injection of an amount of up to $20 \mathrm{ml}$ of contrast agent was performed manually. Otherwise, the automatic injection device was used.

Patients who were not older than 6 years were examined breathing freely, whereas children above the age of 6 received a breathing command. A support cushion was used to ensure a stable position of infants on the moving CT table. In clinical care, diagnostic reading was carried out by board-certified pediatric radiologists.

In $95 \%$ of all examinations, a slice thickness of $3 \mathrm{~mm}$ and an increment of $3 \mathrm{~mm}$ were applied. A slice thickness of $5 \mathrm{~mm}$ and an increment of $5 \mathrm{~mm}$ were chosen in only $5 \%$ of cases. For highresolution $(\mathrm{HR})$ images of the lung parenchyma, a high-resolution kernel (B60f) with a slice thickness of $1 \mathrm{~mm}$ and an increment of $5 \mathrm{~mm}$ was used. Further coronal and sagittal reformations were performed. The typical scan range was chosen from the jugular notch (fossa jugularis sternalis) as the cranial starting point and in the caudal direction including the adrenal glands as a landmark. The mean scan range was $22.9 \mathrm{~cm}$, ranging from $8.6-33.6 \mathrm{~cm}$.

Most examinations were performed with $100 \mathrm{kV}(\mathrm{n}=1104 ; 65 \%)$ or $120 \mathrm{kV}(\mathrm{n}=509 ; 30 \%)$. Less frequent $\mathrm{kV}$ values were $80(n=78$; $4.5 \%)$ and $140 \mathrm{kV}(\mathrm{n}=4 ; 0.5 \%)$. The patients scanned with $140 \mathrm{kV}$ were among the age groups " $11-15$ years" and " $16-17$ years". In these examinations angiography of the thoracic vessels was included, which may contribute to the relatively high dose parameters (effective mAs range: 122 to $128 \mathrm{mAs}$; CTDI $_{\text {vol }}$ range: 11 to $15 \mathrm{mGy})$. Most scans $(\mathrm{n}=1259)$ were non-enhanced. Intravenous contrast agents were used in 436 acquisitions. The injected volume was adjusted to the patients' body weight. 403 of them were primarily contrast-enhanced and 33 examinations included noncontrast and contrast-enhanced series. No iterative reconstruction algorithms were available during the observation time.

\section{Radiation dose}

Tube voltage (kV), volume CT dose index (CTDI; mGy) and dose length product $(\mathrm{DLP} ; \mathrm{mGy} \cdot \mathrm{cm})$ were recorded for all $\mathrm{CT}$ acquisitions and extracted from the examination protocol.

In accordance with the European Guidelines on Quality Criteria for Multislice Computed Tomography, the effective radiation dose $\left(\mathrm{E}_{\text {eff }} ; \mathrm{mSv}\right.$ ) was calculated for all examinations by multiplying DLP with a conversion coefficient, depending on the body region scanned and the patient age (newborns $0.039 ; 1$ year 0.026 ; 5 years $0.018 ; 10$ years $0.013 \mathrm{mSv} /(\mathrm{mGy} \cdot \mathrm{cm})$ ) [23]. The CT scanners used in our study reported a $\mathrm{CTDI}_{\mathrm{vol}}$ referring to a $32-\mathrm{cm}$ body phantom. However, for pediatric patients the conversion coefficient mentioned above refers to a $16-\mathrm{cm}$ body phantom so that our DLP values had to be converted to a $16-\mathrm{cm}$ phantom. For this purpose we used an additional scanner-specific conversion factor provided by the manufacturer ( $\vee$ Table 3 ) to convert DLP to a 
- Table 2 CT devices available in our study.

> Tab. 2 Verfügbare CT-Geräte in der Studie.

\begin{tabular}{|c|c|c|c|c|c|c|}
\hline device & $\begin{array}{l}\text { somatom } \\
\text { sensation } 64\end{array}$ & $\begin{array}{l}\text { somatom } \\
\text { definition flash }\end{array}$ & $\begin{array}{l}\text { somatom } \\
\text { sensation open }\end{array}$ & $\begin{array}{l}\text { somatom } \\
\text { definition }\end{array}$ & $\begin{array}{l}\text { somatom } \\
\text { definition AS+ }\end{array}$ & $\begin{array}{l}\text { somatom } \\
\text { sensation } 16\end{array}$ \\
\hline availability & $05 / 2007-03 / 2014$ & $09 / 2010-03 / 2014$ & $01 / 2007-09 / 2009$ & $01 / 2007-05 / 2010$ & $10 / 2009-07 / 2013$ & $09 / 2008-04 / 2013$ \\
\hline $\begin{array}{l}\text { number of } \\
\text { examinations }\end{array}$ & 716 & 444 & 388 & 124 & 16 & 7 \\
\hline detector & UFC detector & $2 \times$ Stellar detector & UFC detector & UFC detector & UFC detector & UFC detector \\
\hline number of slices & 64 & $2 \times 128$ & 40 & 128 & 128 & 16 \\
\hline rotation time (s) & 0.33 & 0.28 & 0.5 & 0.33 & 0.3 & 0.5 \\
\hline $\mathrm{kV}$ steps $(\mathrm{kV})$ & $80,100,120,140$ & $\begin{array}{l}70,80,100,120, \\
140\end{array}$ & $80,100,120,140$ & $80,100,120,140$ & $\begin{array}{l}70,80,100,120 \\
140\end{array}$ & $80,100,120,140$ \\
\hline max. pitch & 2.0 & 3.2 & 2.0 & 1.5 & 1.5 & 2.0 \\
\hline AEC & CARE Dose4D & CARE Dose4D & CARE Dose4D & CARE Dose4D & CARE kV & CARE Dose4D \\
\hline
\end{tabular}

UFC = Ultra-fast ceramic; AEC = Automatic exposure control, automatische Belichtungssteuerung; $C$ ARE Dose4D = User-specific adjustment of image quality reference $\mathrm{mAs}$ and real-time $\mathrm{mAs}$ modulation for each rotation, benutzerspezifische Anpassung von Bildqualitäts-Referenz-mAs und Echtzeit-mAs-Modulation für jede Rotation; CARE kV= additional automatic kV selection technique, zusätzliche automatische Modulation der Röhrenspannung.

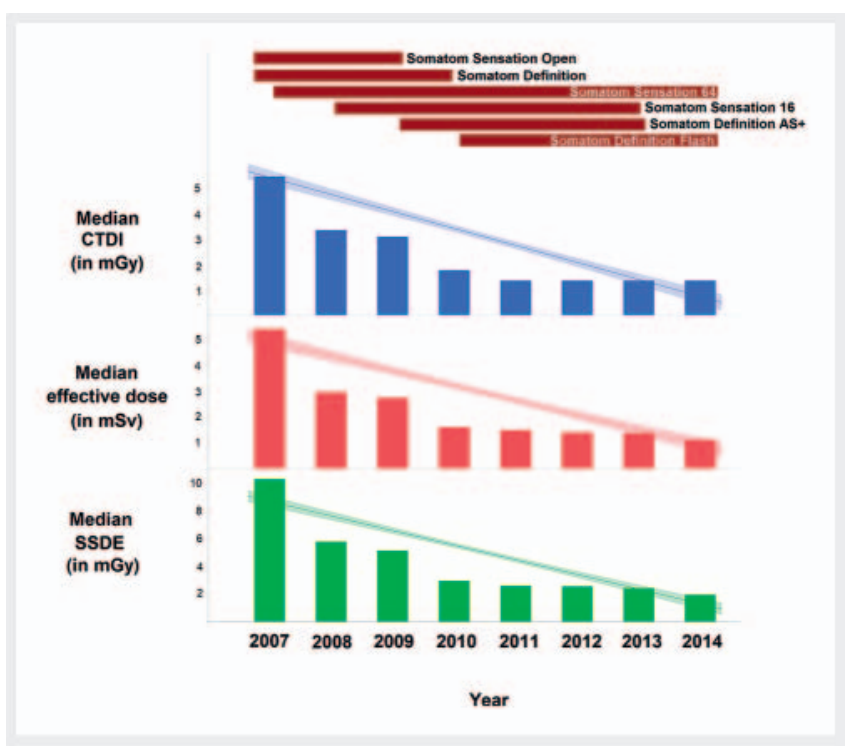

Fig. 2 Dose parameters per year and availability of different scanners. Median volume CT dose index (CTDI; mGy), effective dose (Eeff, $\mathrm{mSv}$ ) and size-specific dose estimate (SSDE; $\mathrm{mGy}$ ) in the different years of the observation time. Linear regression was performed for each parameter and regression bands are shown. Additionally, the period of availability for each scanner is outlined at the top.

- Abb.2 Dosiswerte pro Jahr und Verfügbarkeit der verschiedenen CT-Geräte. Medianwerte für Volumen-CT-Dosisindex (CTDI; mGy), effektive Dosis (Eeff, $\mathrm{mSv}$ ) und size-specific dose estimate (SSDE; mGy) in den verschiedenen Jahres des Beobachtungszeitraums. Es wurde eine lineare Regression mit Angabe der Konfidenzbereiche für jeden Parameter durchgeführt. Zudem ist im oberen Bildabschnitt die zeitliche Verfügbarkeit jedes CT-Gerätes dargestellt. 16-cm phantom suitable for dose estimation in pediatric patients. However, even if the concept of $\mathrm{E}_{\text {eff }}$ has not been developed for patients and its applicability in children is indeed severely limited, it may serve as a rough estimation of the dose delivered.

In accordance with the American Association of Physicists in Medicine, size-specific dose estimates (SSDE; mGy) were calculated for all examinations [24]. Axial images were used to measure the widest transverse and anteroposterior skin-to-skin diameters of each patient and to determine the conversion factor.

To quantify the development of the dose descriptors (CTDI, SSDE, $E_{\text {eff }}$ ) within the observation time, linear regression was performed for each age group. Using the equations of the resulting lines, the percentage reduction in the CTDI, $E_{\text {eff }}$ and SSDE per year was calculated. In addition, median dose parameters were compared between the starting year and the final year of our study for each age group.

\section{High-pitch protocols}

The use of high pitch values was documented in our database. High-pitch protocols were only available on Somatom Definition Flash (Siemens Healthcare GmbH, Forchheim, Germany), which was established in 09/2010 in our institution. Thus, the evaluation of a potential impact of high-pitch protocols on dose estimates was limited to scans within this period ( $\mathrm{n}=944$ scans in total).

\section{Dose modulation technique}

Dose modulation technique was recorded if used. It was available during the entire observation period. Depending on the CT device, an automatic exposure control (AEC) algorithm (CARE Dose 4D) and, where available, an automatic tube voltage adjustment technique (CARE kV; both Siemens Healthcare GmbH, Forchheim, Germany) were used. 
- Table 3 Scanner-specific conversion factors from DLP Ø $32 \mathrm{~cm}$ to DLP Ø $16 \mathrm{~cm}$.

- Tab.3 Scanner-spezifische Konversionsfaktoren von DLP Ø $32 \mathrm{~cm}$ auf DLP Ø $16 \mathrm{~cm}$.

\begin{tabular}{|c|c|c|c|c|c|c|}
\hline pediatric body & $\begin{array}{l}\text { somatom } \\
\text { sensation } 16\end{array}$ & $\begin{array}{l}\text { somatom } \\
\text { definition AS+ }\end{array}$ & $\begin{array}{l}\text { somatom } \\
\text { definition }\end{array}$ & $\begin{array}{l}\text { somatom } \\
\text { definition flash }\end{array}$ & $\begin{array}{l}\text { somatom } \\
\text { sensation open }\end{array}$ & $\begin{array}{l}\text { somatom } \\
\text { sensation } 64\end{array}$ \\
\hline $80 \mathrm{kV}$ & n. a. & n. a. & 2.5 & 2.3 & 2.2 & 2.2 \\
\hline $100 \mathrm{kV}$ & 2.0 & 2.4 & 2.4 & 2.2 & 2.1 & 2.1 \\
\hline $120 \mathrm{kV}$ & 2.0 & 2.3 & 2.3 & 2.2 & 2.1 & 2.0 \\
\hline $140 \mathrm{kV}$ & n. a. & n. a. & n. a. & 2.2 & 2.0 & 2.0 \\
\hline
\end{tabular}

CARE Dose4D enables a user-specific adjustment of "image quality reference $\mathrm{mAs}$ " $\left(\mathrm{mAs}_{\text {ref }}\right)$ for each examination, depending on the required image quality. The parameter expresses the $\mathrm{mAs}$ applied on an average-sized phantom. However, the system also performs real-time mAs modulation for each rotation around the patient, depending on the attenuation profile. For this purpose, a single topogram was performed prior to each scan. Finally, the term of effective $\mathrm{mAs}\left(\mathrm{mAs}_{\mathrm{eff}}\right.$ ) takes the dependence on pitch factor into consideration and is defined as the quotient of $\mathrm{mAs}$ and pitch factor.

In addition, CARE $\mathrm{kV}$ adjusts the tube voltage ( $\mathrm{kV}$ settings) suitable for the patient size and the chosen examination protocol. Tube current is then adapted automatically to maintain a constant contrast-to-noise ratio and to reach an appropriate combination of voltage and current [13]. The two techniques (CARE Dose4D and (ARE kV) can be used simultaneously.

\section{Diagnostic image quality}

To assess the non-inferiority in diagnostic image quality of acquisitions with different radiation dose levels, non-enhanced and i. v. contrast-enhanced examinations $(n=20)$ with the highest and the lowest CTDI in each age group were analyzed. Image quality was assessed independently on a 3-point Likert scale (from 1 = lowest to 3 = highest image quality) by three readers (one senior physicians with seven years of experience and two assistant physicians each with two years of experience) on a standard PACS workstation (Centricity RA 1000, GE Healthcare, Waukesha, Wisconsin, USA). In non-enhanced scans, the pulmonary intersegmental septum of the lower lobe, subsegmental pulmonary arteries and bronchi, as well as motion and breathing artifacts were evaluated. In enhanced scans coronary arteries and sinus, the aortic valve and pulsation artifacts were assessed additionally. Readers were blinded to all identifying data and technical details.

\section{Statistical analysis}

For statistical analysis, IBM SPSS Statistics (version 22 for Windows, Ehningen, Germany) and (SAS jmp, version 11.1.1 for Windows, SAS Institute Inc., Cary, NC, USA) were used.

For all statistical tests, a significance level of $p<0.05$ was set. Continuous variable data are presented as means \pm standard de- viations. Data that did not follow a normal distribution are presented as median with $95 \%$ confidence interval $(\mathrm{Cl})$. By using univariate multifactorial analysis of variance, the influence of age, sex, CT scanner, pitch factor and dose modulation on dose descriptors was evaluated.

A non-inferiority analysis was performed for the 3-point image quality score using a $95 \%$ confidence interval [25]. A non-inferiority margin of 0.2 image quality score points for the difference between the examinations was predefined. To evaluate the interrater reliability, a two-way intraclass correlation coefficient (ICC) was calculated.

\section{Results}

\section{Radiation dose}

Dose descriptors depend on patient age; see the appendix for detailed numerical information about the dose values.

- Fig. 2 demonstrates that the median dose-specific values generally decreased within the observation time. Especially between the years 2009 and 2010, a major leap can be observed. In the period from 2011 to 2013 only minor differences can be found. In addition, Fig. 3 confirms the dose reduction in all age groups. Following linear regression, dose parameters dropped by an average of $12 \%$ per year in the age groups $\leq 1$ year, $2-5$ years, $6-10$ years and $11-15$ years. Only in the age group " $16-17$ years" was a minor annual decrease of $10 \%$ observed. Comparing the median dose values from the starting year 2007 to the final year of our study reveals a percentage decrease of $74 \%$ in the age group $\leq 1$ year and a reduction of $90 \%, 76 \%, 82 \%$ and $82 \%$ in the following age groups.

\section{High-pitch protocols}

In the period from 09/2010 to 03/2014, 414 of 944 examinations were performed with high pitch values $(\geq 3.0)$ on Somatom Definition Flash. - Fig. 4 illustrates the distribution of dose descriptors in the different age groups. All indicators showed lower values when high-pitch protocols were used, independent of contrast agent use. When age, sex, and the use of AEC were included in univariate analysis, these differences between acquisitions with and without high-pitch mode were statistically significant $(p<0.05)$. 


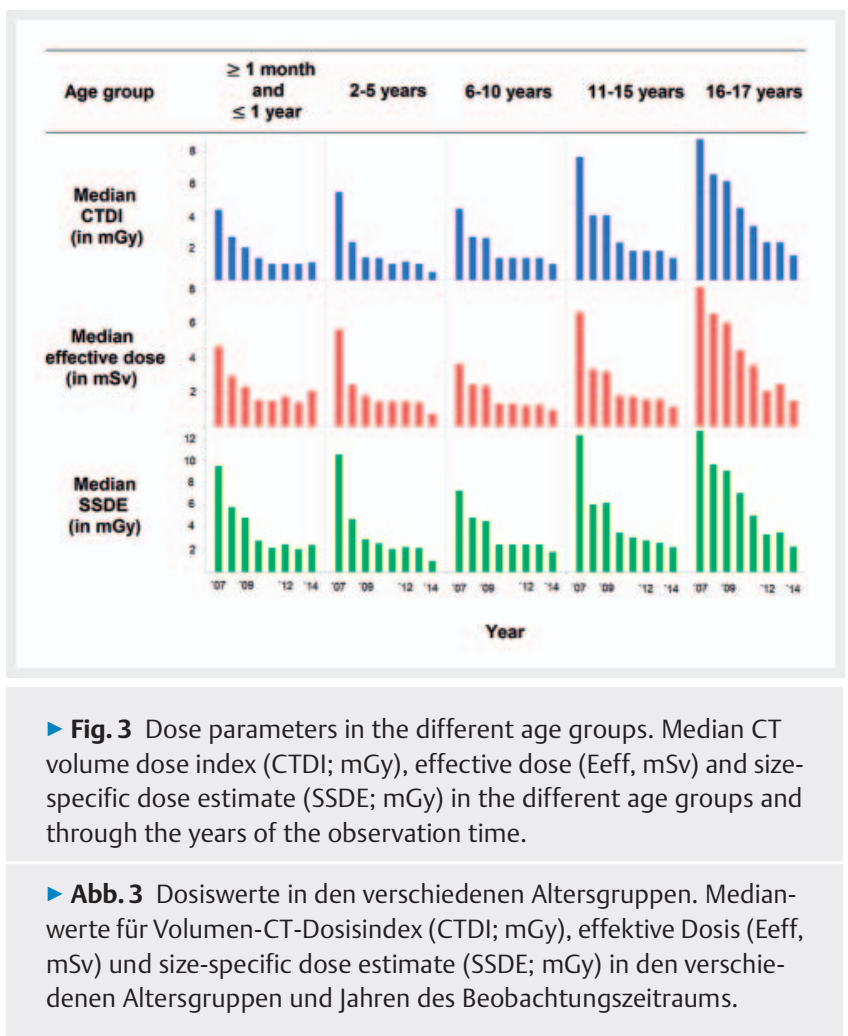

\section{Dose modulation}

A dose modulation technique was used in 409 of all 1695 examinations. These scans were performed with a median effective mAs of $74 \mathrm{mAs}$ [ $95 \% \mathrm{Cl}, 77$; 85]. $80 \mathrm{kV}$ were chosen in 39 cases, $100 \mathrm{kV}$ in 161 scans and $120 \mathrm{kV}$ in 206 scans. Three patients were scanned with $140 \mathrm{kV}$ (Methods and materials, CT examination). These parameters accounted for a median CTDI of $4.3 \mathrm{mGy}[95 \% \mathrm{Cl}, 5.2$; 6.0], a median $\mathrm{E}_{\text {eff }}$ of $4.1 \mathrm{mSv}[95 \% \mathrm{Cl}, 5.1 ; 6.0]$ and a median SSDE of $7.2 \mathrm{mGy}$ [95\% Cl, 8.2; 9.4].

In non-enhanced acquisitions dose parameters were higher in all age groups when AEC was used compared to a manual adjustment of the examination protocol ( $\triangleright$ Fig.5). These differences were statistically significant in the age groups $6-10$ years, 11 15 years and $16-17$ years $(p<0.001)$ - even when sex and the use of different CT devices was taken into account - and not statistically significant in the age groups $\leq 1$ year and $2-5$ years $(p=0.08-0.70)$.

In 298 of 436 contrast-enhanced chest CTs, AEC was carried out. The age groups " $\leq 1$ year" and " $16-17$ years" showed a lower median CTDI, $\mathrm{E}_{\mathrm{eff}}$ and SSDE when AEC was applied. However, only in the age group " $16-17$ years" was the difference statistically significant ( $p=0.04$; age group “ $\leq 1$ year”, $p=0.5)$ ( $>$ Fig. 5 ). The median values of age groups $2-5$ years, $6-10$ years and $11-15$ years presented higher dose parameters in scans with dose modulation. For the two older age groups, these differences were statistically significant ( $p<0.001$; age group “ $2-5$ years”, $p=0.2$ ).

\section{Image quality}

The mean image quality scores were 2.18 [95\% Cl, 2.04; 2.32$]$ in the subgroup with the highest CTDI values and $2.14[95 \% \mathrm{Cl}$,
1.98; 2.30] in acquisitions with the lowest radiation dose. Thus, differences in image quality between the examinations with the highest and lowest CTDI values resided within the predefined non-inferiority margin ( $\triangleright$ Fig. 6 ). The overall inter-rater reliability was found to be ICC 0.889, (95\% Cl: 0.784, 0.950; $p<0.001)$, indicating almost perfect agreement.

\section{Discussion}

In our retrospective observational study over a period of seven years, a significant reduction in radiation dose in pediatric chest CT was observed without a loss of diagnostic image quality using state-of-the-art scanners and dose optimization strategies. In particular, the implementation of high-pitch scanning seems to essentially contribute to this development. Interestingly in this context, the different AEC methods provided were not as efficient as expected. In an early patient study dose values were reduced by an average of $38 \%$ when using tube current modulation [14].

CT scanners and software equipment evolved as decisive influencing factors of CT radiation dose [19]. Meanwhile the focus shifted from high-quality $\mathrm{CT}$ imaging with precise anatomic information to better manage radiation dose and to maintain a diagnostic image quality related to the clinical question. However, this fact should be further addressed in national and international guidelines for radiation protection. During the process of accepting noisier images without reducing diagnostic capabilities, pediatric imaging has adopted a pioneering role [7]. Even with regard to adult $\mathrm{CT}$, a maximum of image quality is rarely required to answer the clinical question.

Comparing our dose parameters (Appendix) to a national survey from 2007 that proposed reference dose values in pediatric CT for each age group, we found that in the initial year of the observation time (2007) the median CTDI in our study was at the upper limit of the corresponding reference values [26], for example in children aged $2-5$ with a median CTDI of $5.4 \mathrm{mGy}$ and a reference value of $5.5 \mathrm{mGy}$. In this period, the CTDI exceeded the reference level in the age group of infants with only four patients $(\geq 1$ month and $\leq 1$ year; median CTDI, 4.3 mGy; reference value 3.5 mGy). However, the CTDI was clearly below the reference levels in the following years across all age groups, also compared to the latest announcement of the Federal Office for Radiation Protection (BfS) [27]. Especially in the last years of the observation time (2013 and 2014), the CTDI was more than three times lower than the corresponding reference value in some age groups, for example in children aged $11-15$ with a median CTDI of $1.8 \mathrm{mGy}$ (reference value, $6.5 \mathrm{mGy}$ ) matching a range of low-dose imaging.

Miglioretti et al. reported mean radiation doses of 744 scans performed between 2001 and 2011 that were more than twice as high as in our study, when comparing the corresponding age groups [1]. It can be supposed that different local guidelines for radiation protection and the technical developments made since the ending of this previous study account for the differences in dose estimation. However, it has to be considered that iterative reconstruction was not applied in our study and its use may have led to a further dose reduction [16]. 


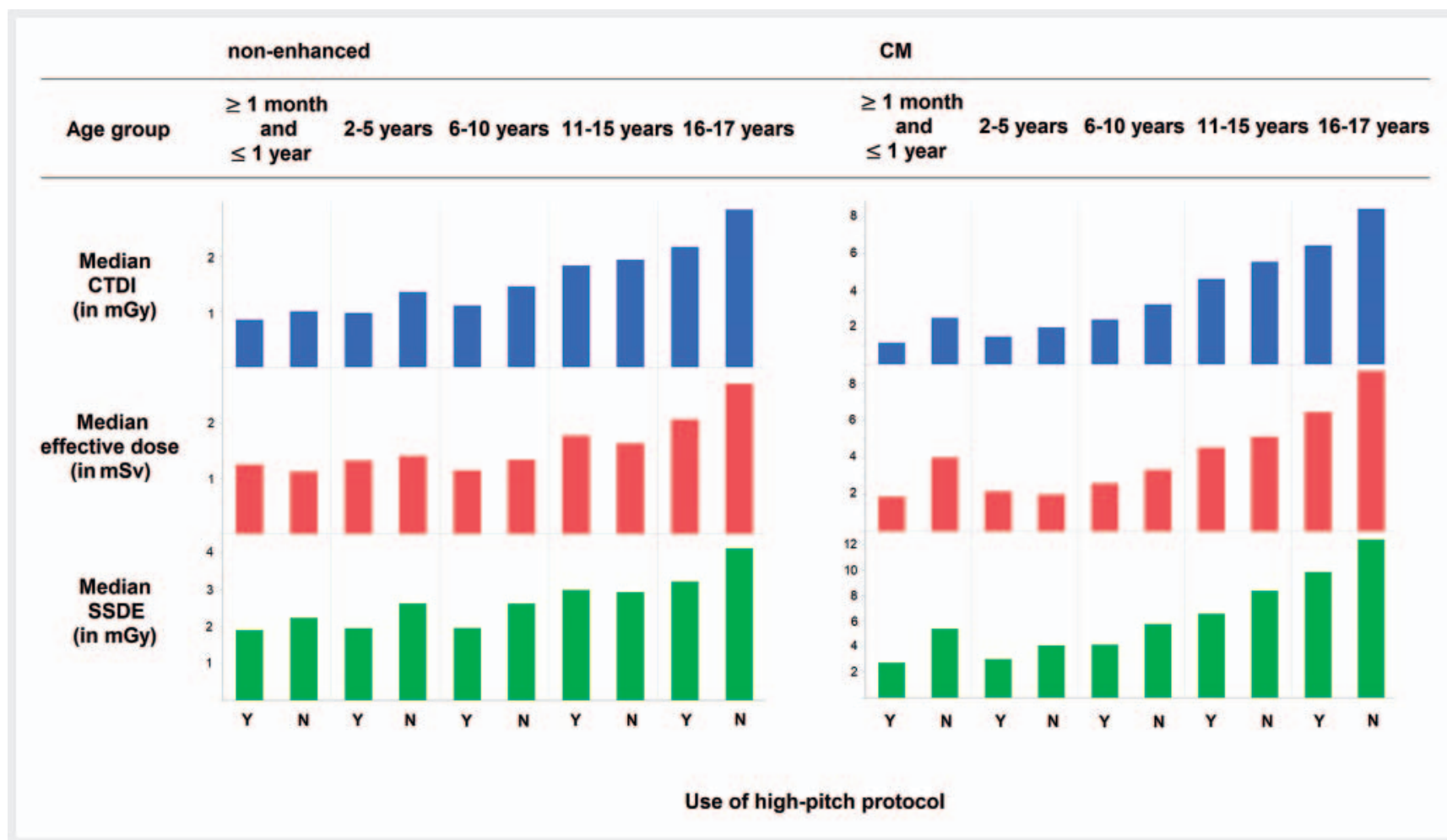

- Fig. 4 Dose parameters depending on the application of high-pitch mode. Median volume CT dose index (CTDI; mGy), effective dose (Eeff, mSv) and size-specific dose estimate (SSDE; $\mathrm{mGy}$ ) in the different age groups depending on the application of high-pitch mode $(\mathrm{Y}=\mathrm{yes}, \mathrm{N}=\mathrm{no})$ and contrast agent use (left, non-enhanced; right CM = contrast agent). This figure is limited to scans in the period from 09/2010 to 03/2014 ( $n=944$ ).

- Abb. 4 Dosiswerte in Abhängigkeit von Verwendung des High-pitch-Modus. Medianwerte für Volumen-CT-Dosisindex (CTDI; mGy), effektive Dosis (Eeff, $\mathrm{mSv}$ ) und size-specific dose estimate (SSDE; $\mathrm{mGy}$ ) in den verschiedenen Altersgruppen in Abhängigkeit der Verwendung des HighPitch-Modus ( $\mathrm{Y}=$ ja, $\mathrm{N}=$ nein) und einer Kontrastmittelapplikation (links, nativ; rechts, kontrastangehoben). Die Abbildung beschränkt sich auf die Untersuchungen in der Zeitspanne von 09/2010 bis 03/2014 ( $\mathrm{n}=944)$.

The implementation of dual-source CT devices allowed for high-pitch scanning, which has been applied to pediatric chest and cardiac CT to reduce motion artifacts [7]. Our data included a significant decline in median dose values from 2009 to 2010, when Siemens Somatom Definition Flash (Siemens Healthcare $\mathrm{GmbH}$, Forchheim, Germany) with the possibility of high-pitch protocols was implemented. Providing a large patient population, the method appears recommendable in all age groups and independent of contrast agent use. In addition, further settings of the scanner may have contributed to dose reduction: In contrast to the other CT devices in our study, Somatom Definition Flash has two detectors combining the photodiode and an analog-to-digital converter, which may reduce electronic noise, especially in lower dose applications ( $\vee$ Table 2 ). In smaller patient populations and compared to our patient cohort - restricted age groups, favorable radiation doses have been reported with the high-pitch technique for pediatric chest CT imaging and CT angiography (CTA) [18, 28, 29]. In addition, several previous studies have suggested that high-pitch scanning does not compromise image quality, which seems reasonable in light of our results $[28,29]$.

Our data demonstrate that the use of a dose modulation technique was not always advantageous and should be considered individually. Karmazyn et al. found that in abdominal CT with AEC, dose reduction was least effective for body weight less than $20 \mathrm{~kg}$ (our age groups $1-3$, considering Child Growth Standards of the World Health Organization, WHO) [30, 31]. The vast majority of children from ages $2-15$ received higher radiation exposure when AEC was used in contrast-enhanced chest CT. However, adolescents (16 and 17 years) seem to benefit from dose modulation, which agrees with Karmazyn's statement about the highest effectiveness of AEC at a body weight range over $60 \mathrm{~kg}$ [30]. However, according to our results, dose modulation is applicable in infants, which contradicts the previous findings. One of the reasons for this phenomenon could be that manual adjustment is hampered in the youngest and oldest age groups, so that the stored algorithms of AEC achieve comparatively favorable dose parameters. In infants a relatively low patient number and little experience with manual adaption in the early years of the study can be considered as possible reasons. However, the oldest age group presents a rather heterogeneous patient cohort concerning weight and body diameter, so that fluctuating dose values may occur. Our results are important because radiologists should not only take patient size but also the use of contrast agent into account when considering pediatric chest CT with dose modulation.

The use of dose modulation systems includes potential sources of error, which may explain the heterogeneous data in the litera- 


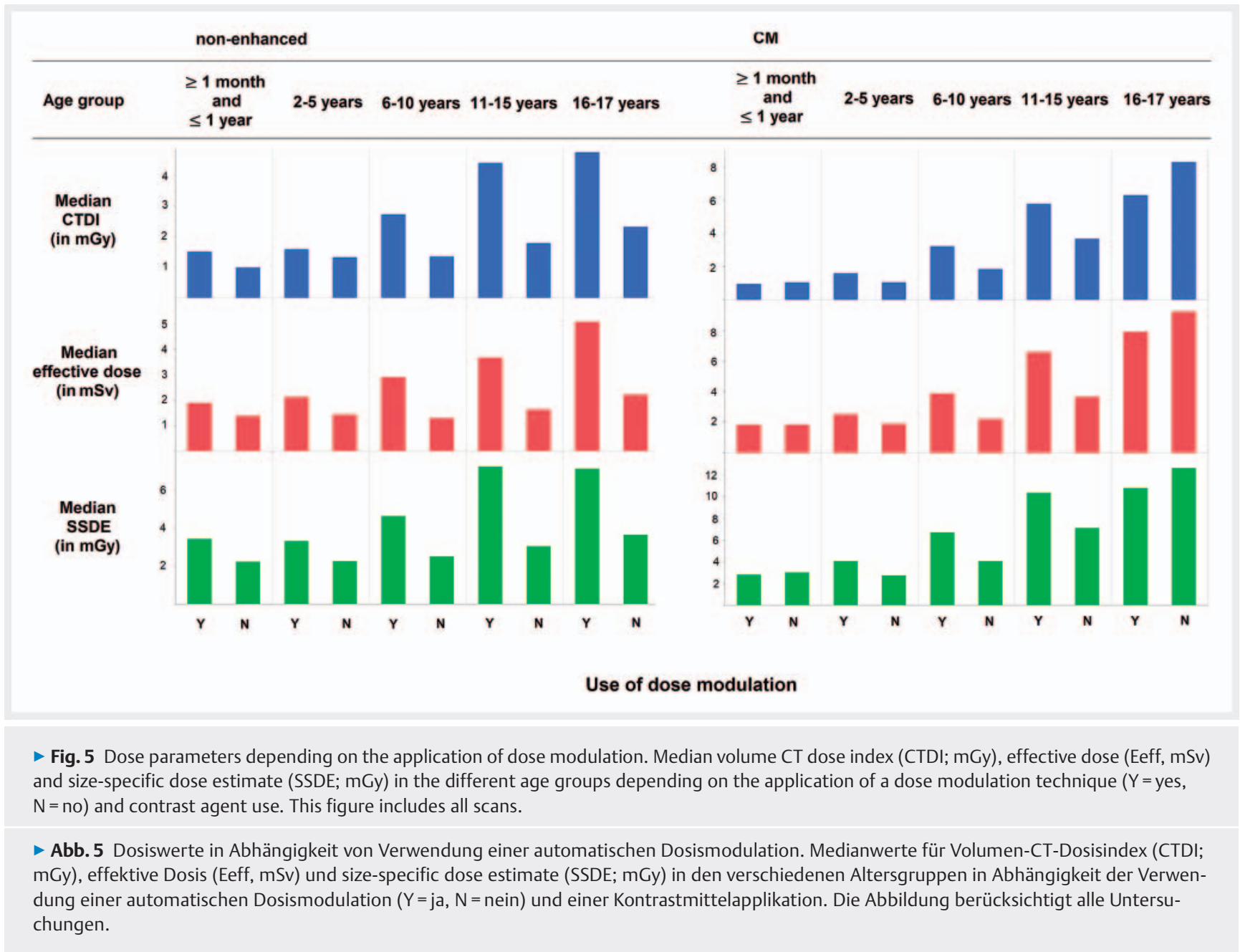

ture and the unfavorable dose parameters in our study. A possible technical problem refers to an inappropriate configuration of the scanner for the use of AEC with different patient sizes. In this context, we suspect that the expertise of the manufacturer and our experience with AEC protocol utilization were limited in the early years of our study. At that time, it is possible that inappropriately high reference $\mathrm{mAs}$ values were selected, which may partly explain the elevated dose values and provides some potential for further optimization. After AEC protocols were adopted from the manufacturer initially, they have been adjusted in the following years with increasing know-how and growing feedback to the manufacturer. In addition, patient movement between the localizer and subsequent scans may lead to application of an inappropriately adjusted tube current profile. Finally, an inaccurate position on the CT table and insufficient centering of the patient may be relevant in younger children [32]. In this context, manual adjustment should be considered as a reasonable alternative to $A E C$, at least in dedicated pediatric centers with the necessary pediatric CT experience. Further development on this issue for the pediatric population is recommended.

Some aspects of our study may seem obvious. Nevertheless such a calculation of dose values and analysis of the dose development in the context of the clinical routine process have not yet been published to the best of our knowledge. As low $\mathrm{kV}$ protocols and iterative reconstruction are not universally included in pediatric CT today, the results of our study still seem relevant for a majority of pediatric examinations worldwide.

Our study has the following limitations: The study was set up at a single center; consequently it depends on the local standard of care and the on-site technical installations, for example CT devices of only a single manufacturer were used. Additionally, due to the lack of availability, iterative reconstruction algorithms and low $\mathrm{kV}$ protocols were not included.

In summary, radiation dose in pediatric chest CT has been considerably reduced in the last decade without a relevant loss of image quality. High-pitch scanning appears to be an effective method of dose optimization whenever iterative reconstruction is not available. However, in our institution the use of dose modulation techniques had to be considered individually according to patient size and depending on the examination protocol. In this context, a centralized position of the patient on the CT table is of basic importance for reliable dose adjustment. Moreover, additional options are available today to tap the further potential of dose reduction, for example iterative reconstruction. 


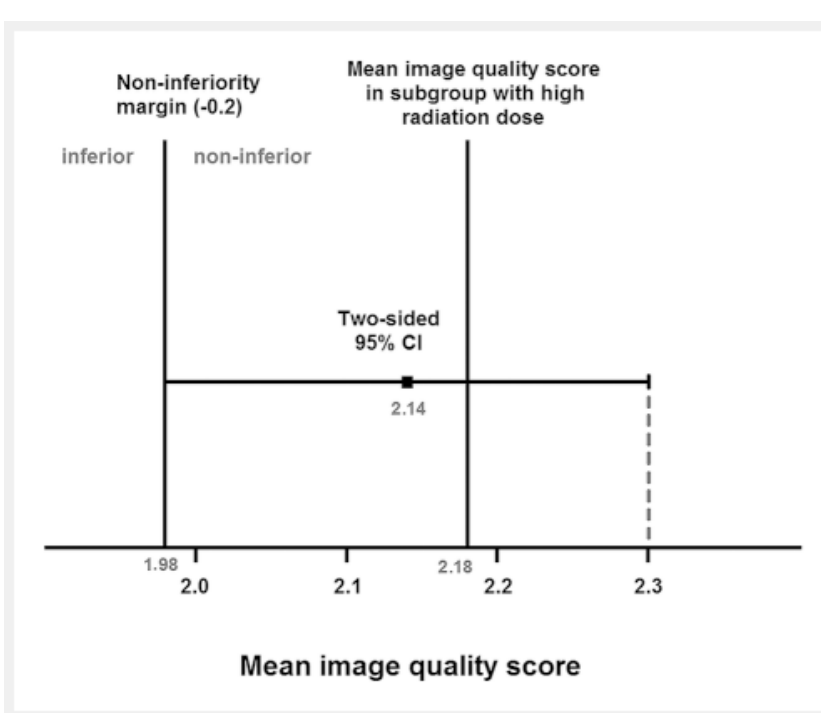

- Fig. 6 Non-inferiority analysis of image quality scores. Non-inferiority analysis with the mean image quality score of the subgroup with high radiation dose (standard method; 2.18 ) and the resulting non-inferiority range $(2.18-1.98)$. Two sided $95 \%$ confidence interval $(\mathrm{Cl})$ of the test method (subgroup with low radiation dose; $\mathrm{Cl}$ $95 \%, 1.98 ; 2.30)$ resides within the margin.

- Abb. 6 „Non-inferiority“-Analyse der Bildqualität. „Non-inferiority"-Analyse mit Darstellung des mittleren Punktwertes der Bildqualität in der Untergruppe mit hoher Strahlendosis (Standardmethode; 2.18 ) und des entsprechenden „Non-inferiority“-Bereichs $(2.18-1.98)$. Das zweiseitige $95 \%$-Konfidenzintervall (Cl) der Testmethode (Untergruppe mit niedriger Strahlendosis; Cl 95\%, 1.98; 2.30) verbleibt innerhalb dieser Grenzen.

\section{CLINICAL RELEVANCE}

- High-pitch protocols should be used for pediatric chest CT.

- Acquisitions with fixed parameters should be considered as an alternative to AEC.

- The use of AEC may be advantageous in adolescents.

$\begin{array}{ll}\text { ABBREVIATIONS } \\ \text { AEC } & \text { Automatic Exposure Control } \\ \text { CI } & \text { Confidence Interval } \\ \text { CT } & \text { Computed Tomography } \\ \text { CTA } & \text { Computed Tomography Angiography } \\ \text { CTDI } & \text { Volume CT Dose Index } \\ \text { DLP } & \text { Dose Length Product } \\ \text { Eff }_{\text {eff }} & \text { Effective Radiation Dose } \\ \text { ICC } & \text { Intraclass Correlation Coefficient } \\ \text { IV } & \text { Intravenous } \\ \text { SSDE } & \text { Size-Specific Dose Estimate } \\ \text { WHO } & \text { World Health Organization }\end{array}$

\section{Conflict of Interest}

The authors declare that they have no conflict of interest.

\section{References}

[1] Miglioretti DL, Johnson E, Williams A et al. The use of computed tomography in pediatrics and the associated radiation exposure and estimated cancer risk. JAMA Pediatr 2013; 167: 700-707

[2] Journy N, Ancelet S, Rehel JL et al. Predicted cancer risks induced by computed tomography examinations during childhood, by a quantitative risk assessment approach. Radiat Environ Biophys 2014; 53: 39-54

[3] European Society of Radiology (ESR). White paper on radiation protection by the European Society of Radiology. Insights Imaging 2011; 2: 357-362

[4] Berrington de González A, Mahesh M, Kim KP et al. Projected cancer risks from computed tomographic scans performed in the United States in 2007. Arch Intern Med 2009; 169: 2071-2077

[5] Linet MS, Kim KP, Rajaraman P. Children's exposure to diagnostic medical radiation and cancer risk: epidemiologic and dosimetric considerations. Pediatr Radiol 2009; 39 (Suppl. 1): S4-S26

[6] Nievelstein RA, van Dam IM, van der Molen A). Multidetector CT in children: current concepts and dose reduction strategies. Pediatr Radiol 2010; 40: 1324-1344

[7] Valentin J (Editor). The 2007 Recommendations of the International Commission on Radiological Protection ICRP publication 103. Ann ICRP 2007; 37: 1-332

[8] Pearce MS, Salotti JA, Little MP et al. Radiation exposure from CT scans in childhood and subsequent risk of leukaemia and brain tumours: a retrospective cohort study. Lancet 2012; 380: 499-505

[9] Mathews JD, Forsythe AV, Brady Z et al. Cancer risk in 680000 people exposed to computed tomography scans in childhood or adolescence: data linkage study of 11 million Australians. BMJ 2013; 346: f2360

[10] Krille L, Dreger S, Schindel R et al. Risk of cancer incidence before the age of 15 years after exposure to ionising radiation from computed tomography: results from a German cohort study. Radiat Environ Biophys 2015; 54: 1-12

[11] Kalender WA, Buchenau S, Deak P et al. Technical approaches to the optimisation of CT. Phys Med 2008; 24: 71 - 79

[12] Zheng M, Zhao H, Xu J et al. Image quality of ultra-low-dose dual-source $\mathrm{CT}$ angiography using high-pitch spiral acquisition and iterative reconstruction in young children with congenital heart disease. J Cardiovasc Comput Tomogr 2013; 7: 376-382

[13] Kok M, Mihl C, Seehofnerova A et al. Automated Tube Voltage Selection for Radiation Dose Reduction in CT Angiography Using Different Contrast Media Concentrations and a Constant lodine Delivery Rate. Am J Roentgenol 2015; 205: 1332-1338

[14] Greess H, Wolf H, Baum U et al. Dosage reduction in computed tomography by anatomy-oriented attenuation-based tube-current modulation: the first clinical results. Rofo 1999; 170: 246-250

[15] Braun FM, Johnson TR, Sommer WH et al. Chest CT using spectral filtration: radiation dose, image quality, and spectrum of clinical utility. Eur Radiol 2015; 25: $1598-1606$

[16] den Harder AM, Willemink MJ, Budde RP et al. Hybrid and model-based iterative reconstruction techniques for pediatric CT. Am J Roentgenol 2015; 204: 645-653

[17] Lell MM, May M, Deak P et al. High-pitch spiral computed tomography: effect on image quality and radiation dose in pediatric chest computed tomography. Invest Radiol 2011; 46: 116-123

[18] Tsiflikas I, Thomas C, Ketelsen D et al. High-pitch computed tomography of the lung in pediatric patients: an intraindividual comparison of image 
quality and radiation dose to conventional 64-MDCT. Rofo 2014; 186 : $585-590$

[19] McCollough CH, Primak AN, Braun N et al. Strategies for reducing radiation dose in CT. Radiol Clin North Am 2009; 47: 27 - 40

[20] Remy-Jardin M, Santangelo T, Colas L et al. Radiation dose levels in pediatric chest CT: experience in 499 children evaluated with dual-source single-energy CT. Pediatr Radiol 2017; 47: 161 - 168

[21] Ogden CL, Kuczmarski RJ, Flegal KM et al. Centers for Disease Control and Prevention 2000 growth charts for the United States: improvements to the 1977 National Center for Health Statistics version. Pediatrics 2002; 109: 45-60

[22] Veit R, Guggenberger R, Nosske D et al. Diagnostic reference levels for X-ray examinations: update 2010. Radiologe 2010; 50: 907 - 912

[23] Bongartz D, Golding SJ, Jurik AG et al. European Guidelines for Multislice Computed Tomography, Funded by the European Commission, Contract number FIGM-CT2000-20078-CT-TIP. 2004

[24] American Association of Physicists in Medicine Report No. 204. SizeSpecific Dose Estimates (SSDE) in Pediatric and Adult Body CT Examinations. College Park, MD, 2011

[25] Ahn S, Park SH, Lee KH. How to demonstrate similarity by using noninferiority and equivalence statistical testing in radiology research. Radiology 2013; 267: $328-338$
[26] Galanksi M, Nagel HD, Stamm G. Pädiatrische Expositionspraxis in der Bundessrepublik Deutschland. Ergebnisse einer bundesweiten Umfrage 2005/06. Fortschr Röntgenstr 2007; 179: 1110-1111

[27] Federal Office for Radiation Protection (BfS). Publication of updated diagnostic reference levels for diagnostic and interventional X-ray examinations. 2016.:4,Tab. 8

[28] Achenbach S, Marwan M, Ropers D et al. Coronary computed tomography angiography with a consistent dose below $1 \mathrm{mSv}$ using prospectively electrocardiogram-triggered high-pitch spiral acquisition. Eur Heart J 2010; 31: 340-34627

[29] Esser M, Gatidis S, Teufel M et al. Contrast-Enhanced High-Pitch Computed Tomography in Pediatric Patients Without Electrocardiography Triggering and Sedation: Comparison of Cardiac Image Quality With Conventional Multidetector Computed Tomography. J Comput Assist Tomogr 2017; 41: $165-171$

[30] Karmazyn B, Ai H, Liang $Y$ et al. Effect of body size on dose reduction with longitudinal tube current modulation in pediatric patients. Am J Roentgenol 2015; 204: 861 - 864

[31] The WHO Child Growth Standards (2017, March 18). Retrieved from http://www.who.int/childgrowth/standards/en/

[32] Cody DD. Management of auto exposure control during pediatric computed tomography. Pediatr Radiol 2014; 44 (Suppl. 3): 427 -430 